\title{
Osteopathic Approach to Diagnosis and Treatment of Dysfunction at the Thoracolumbar Junction
}

Drew D. Lewis, DO

Financial Disclosures: None reported.

Support: None reported.

Address correspondence to

Drew D. Lewis, DO,

Des Moines University College

of Osteopathic Medicine, 3200

Grand Ave, Des Moines, IA

50312-4104.

Email address:

drew.d.lewis@dmu.edu

Submitted

April 8, 2019;

accepted

May 8, 2019.

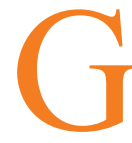
ordon Zink, DO, presented an efficient and effective means of approaching a patient from the respiratory circulatory model with a goal of maximizing venous, lymphatic, and arterial flow to improve health. ${ }^{1}$ Some of the key somatic dysfunctions in his treatment approach were found at the transition zones of the body, which are not only areas of increased stress, but are also closely associated with transverse diaphragms. ${ }^{1}$

At the thoracolumbar junction (T12-L1), the kyphotic curve of the thoracic spine is transitioning to the lordotic curve of the lumbar spine. This transition zone is therefore a crossover or inflection point of the spine and is subject to increased stresses, injury, and somatic dysfunctions. The horizontal diaphragm associated with the thoracolumbar region of the spine is the thoracoabdominal diaphragm.

The thoracoabdominal diaphragm has widespread attachments throughout the region, including to the lower 6 ribs, T12, L1-2 or 3, and the xyphoid process of sternum. ${ }^{2}$ Key myofascial relationships exist from the mediastinum through the diaphragm and its crus and down into the pelvis and lower extremity through the quadratus lumborum and psoas muscles.

Somatic dysfunction in the region can affect the pump mechanism of the thoracoabdominal diaphragm and contribute to venous and lymphatic stasis in the trunk and extremities. ${ }^{3}$ The thoracoabdominal diaphragm is pumplike with regard to drawing oxygenated air into the lungs. The negative intrathoracic pressure created during inhalation greatly assists in venous and lymphatic return to the central circulation. ${ }^{1}$

Viscerosomatic relationships from the lower intestine and colon and other abdominopelvic organs exist with the sympathetic centers in the thoracolumbar region. ${ }^{4}$ These fascial or articular somatic dysfunctions can lead to restriction or pain.

Zink presented an efficient means of evaluating gross fascial pattern movements associated with the transition zones, helpful in identifying both fascial or articular restrictions. With the diagnosis and treatment of somatic dysfunction of the thoracolumbar region, it is necessary to consider the entire thoracoabdominal cylinder.

Gross fascial motion of the thoracic cage on the lumbar spine can be assessed in a supine patient with the thoracolumbar shift of the lower thorax. It is also helpful to assess lumbar sidebending with static position evaluation of which the iliac crest is more superior. Thoracolumbar somatic dysfunction treatment addresses both the thoracolumbar shift and the superior iliac crest.

The patient is placed in a lateral recumbent position on the side opposite of the thoracolumbar shift. To isolate motion, the patient's trunk is rotated toward the ceiling until motion is first appreciated at the T12 vertebra. Monitoring hands can be switched. The trunk is stabilized by having the patient wrap his or her arms around the physician's arm. The patient's leg adjacent to the table is extended. The contralateral leg (not positioned on the table) is flexed at the hip (allowing the leg to drop off the table) until motion is first appreciated at T12. The physician stabilizes the patient's upper body with one arm, and with the other arm on the lateral pelvis region, the patient's lumbar spine and pelvis are rotated forward toward the physician and either slightly superior or inferior to directly address the positional diagnosis of the iliac crest. As the patient exhales, the lumbar spine and pelvis are further rotated forward (toward the table) to fully engage the restrictive barrier. A highvelocity, low-amplitude thrust is performed only with the arm on the pelvis, directing the vector toward the physician (further rotating the pelvis 
forward) and slightly superior (if the iliac crest was inferior) or slightly inferior (if the iliac crest was superior).

While correction is often assumed when a joint articulation, or "pop," is appreciated, a (direct) myofascial improvement can also be achieved with or without an articulation. The thoracolumbar shift and iliac crest height can be reassessed to evaluate the effectiveness of the treatment. The complex nature of the structure and function of the thoracolumbar junction has lent itself to many different diagnostic and treatment approaches. This can be evidenced by looking at the multitude of approaches for treatment of the thoracoabdominal diaphragm.

The technique presented in the video is effective for the treatment of somatic dysfunction of the thoracolumbar junction. Treating this region may help reduce postural compensatory mechanisms, balance sympathetic nervous system through viscerosomatic reflexes, improve gait, and decrease pain. This technique is effective in improving venous and lymphatic return; however, it is best integrated as part of a whole-body respiratory-circulatory model approach. ${ }^{3,5}$

The benefits can be especially valuable during pregnancy, not only for common pregnancy-related back pain, but also for the restriction of maximal respiration. The respiratory-circulatory approach can be particularly helpful for reducing the effects of congestion-related conditions seen in pregnancy (eg, carpal tunnel syndrome, varicosities, hemorrhoids, and pedal edema). ${ }^{1,3,6}$

By also focusing on the remaining transverse diaphragms in addition to treatments of other key somatic dysfunctions that may inhibit venous, lymphatic, and arterial flow, Zink's common compensatory pattern approach is truly a model that promotes health. (doi:10.7556/jaoa.2020.023)

\section{Acknowledgments}

We thank Eric Fishback for his video editing and Amber Brown, OMM Fellow, OMS V, for being the patient model in the video.

\section{References}

1. Kuchera ML. Lymphatics approach. In: Chila AG, executive ed. Foundations of Osteopathic Medicine. 3rd ed. Lippincott Williams \& Wilkins; 2011:787-807

2. DeStefano L, ed. Greenman's Principles of Manual Medicine. 4th ed Philadelphia, PA: Lippincott Williams \& Wilkins; 2011:9.

3. Zink JG, Lawson WB. Pressure gradients in the osteopathic manipulative management of the obstetric patient. Osteopath Ann. 1979;7(5):208-214.

4. Willard FH. Autonomic nervous system. In: Chila AG, executive ed. Foundations of Osteopathic Medicine. 3rd ed. Lippincott Williams \& Wilkins; 2011:154.

5. Zink JG. Respiratory and circulatory care: the conceptual model. Osteopath Ann. 1977;5(3):108-112.

6. Tettambel M. Lower extremity swelling in pregnancy. In: Chila AG, executive ed. Foundations of Osteopathic Medicine. 3rd ed. Lippincott Williams \& Wilkins; 2011:963.

๑) 2020 American Osteopathic Association 